\title{
Leveraging the Cultural Model for Opportunistic Networking in sub-Saharan Africa
}

\author{
Jonathan Ouoba ${ }^{1,2}$ and Tegawendé F. Bissyandé ${ }^{1,2}$ \\ 1 The FasoLabs Project, Burkina Faso \\ 2 LaBRI, University of Bordeaux, France \\ \{jonathan.ouoba, tegawende.bissyande\}@fasolabs.org
}

\begin{abstract}
The immense potential of ICT for improving users' livelihood has been discussed in a large body of literature and many instantiations in our daily life demonstrate this reality. In developing areas, such as Sub-Saharan Africa, ICT for development has become the frontrunner initiative that decision makers are pushing to bring millions of people out of poverty. Unfortunately, the majority of Africans, who live in rural areas, fail to identify with the existing various solutions.

We propose the Toolé approach, which aims at vulgarizing new technologies for facilitating and automating the collection and synthesis of agricultural information. The originality of Toole lies in the fact that it attempts to build on the cultural values of peasants. The architecture on top of which Toolé was built relies on cheap, yet powerful, devices and on readily available peer-to-peer protocols to deliver services whose inherent costs make sense for low income areas.
\end{abstract}

Key words: Toolé, ICT4D, sub-Saharan Africa, remote areas, e-agriculture, opportunistic networking, cultural phenomenon

\section{Introduction}

Despite a steady progress in the last decade, sub-Saharan Africa still lags behind the rest of the world in terms of access to the information society. According to a 2011 report of the International Telecommunication Union, the average Internet penetration rates in the area are less than $12 \%$ while the world average is $30 \%$ with a $80 \%$ rate in developed areas [7]. In last ranked countries such as Burkina Faso, where the Internet penetration rate is as low as $1 \%$, only around $0.2 \%$ of households have Internet access. Furthermore, in rural regions, the phenomenon is even more noticeable because of the limited infrastructure. This situation is not expected to evolve, at least not in the foreseeable future, as the priorities of the central government as well as of the local populations lie elsewhere. Actually, investing in telecommunication infrastructure will bring out social inconsistencies since the lack of health care centers, schools, and practicable roads has more damaging impacts on the communities.

Nevertheless, in Burkina Faso for example, about $85 \%$ of the workforce live on agricultural activities in rural areas. While communities have been mostly 
engaged in subsistence farming, the revenues generated by commercial agriculture, with internationally demanded crops such as sugarcane and cotton, have incentivised peasants to produce for regional, national and international markets. Unfortunately, sub-Saharan farmers have limited means and must regroup in cooperatives that often require logistic support from central and local government officials. One example of such support is communication capabilities for disseminating strategic information to all members of the cooperative. These capabilities mostly consist of on-road transportations of cooperative representatives through scattered camps, villages and agricultural fields, to hold informative meetings. This schema, however, raises numerous issues, the most salient of which are : (1) the regularity of the information in a world where e.g., feedstock prices fluctuate daily, if not hourly; the autonomy of cooperatives when financial and natural constraints, such as a bridge collapse during the rainy season, prevent the holding of a meeting; 3 the possibility for a horizontal cooperation between cooperative members outside planned meetings; and $\boldsymbol{\Phi}$ the complexity/pertinence of the information transmitted when illiteracy is commonplace.

An alternate communication scheme could rely on the local peer-to-peer networking opportunities that arise when cooperative members carrying mobile devices meet and go their ways. This scheme allows to harness the potential of their many devices which can be interconnected in a P2P system for spreading information. Such a system is furthermore self-scalable and naturally balanced, since members can forward as well as retrieve information data [16], and is more suited to the context of rural areas, where farmers are mobile through agricultural fields, and through markets across neighboring villages.

This paper. In this paper we propose to leverage the cultural model in sub-Saharan Africa to build a collaborative system for exchanging complex data among cooperative members scattered in different villages. In our approach, hereafter referred to as Toolé, ${ }^{1}$ an opportunistic networking strategy allows to seamlessly propagate a piece of information while sidestepping the difficulties encountered in existing scenarios.

The main contributions of this paper are as follows:

$\checkmark$ We propose an approach to cooperative-level communication of strategic information data based on opportunistic networking scenarios.

$\checkmark$ We discuss the opportunity for our approach by revisiting the challenges in collecting, computing and conveying strategic information to and within remote rural areas.

$\checkmark$ We demonstrate the suitability of opportunistic networking solutions in subSaharan Africa through an assessment based on different criteria established in existing literature.

\footnotetext{
1 Toolé refers to an object or/and a message that a given person is asked to deliver to a third party on behalf of someone else. The word is borrowed from mòoré, a major native language of Burkina Faso, and is part of a culture where people by solidarity assist each other in forwarding one's news to his distant relatives or conveying commissions of goods.
} 
$\checkmark$ We also propose a network architecture based on affordable technologies such as Bluetooth for local P2P communications and NFC for configuring and automating these communications.

The rest of this paper is organized as follows: Section 2 discusses the relevance of ICT in the process of disseminating information in rural areas and the associated challenges. Section 3 presents the context of sub-Saharan Africa with its challenges as well as the cultural facts that are leveraged in this work. Section 4 presents our approach through a real-world application scenario and details the network entities involved as well as a snapshot overview of the algorithms. Section 5 discusses related work and Section 6 concludes.

\section{Using ICT in sub-Saharan rural areas}

In recent years the focus has shifted on the fight against rural poverty as a sound strategy for hastening development in third-world countries. ICT has then been relied upon to support governments as well as NGOs' initiatives that were directed at finding ways to improve peasants' incomes. In this context, researchers and practitioners alike have shown a keen interest to e-agriculture, which involves the use of ICT for rural development, with a primary focus on sustainable agriculture. One of the main questions in the field is how to effectively provide useful and reliable information to farmers in remote areas. While many projects have proposed various innovations with relative success, we believe that the situation is still unfavorable in many parts of Sub-Saharan Africa because general-purpose off-the-shelf solutions attempt to make users accommodate with the products instead of exploiting properties of the deployment context. Before diving into the description of the different facets of our approach, we revisit in this section the challenges that arise when attempting to build and disseminate "meaningful" information within rural areas.

Useful Information : From What and For Whom? A basic research question that arises in the implementation of ICT solutions for rural areas is : what types of complex information do farmers need for the prosperity of their activities? Unfortunately, there appears to be no clear vision of how the agricultural sector should develop in terms of ICT adoption in maximizing benefits for the farmer community [6]. Besides, collecting and disseminating information to improve agricultural steps is a tedious endeavor as service providers must be able to determine real user needs. Finally, because coordination of professionals is improbable in sub-Saharan Africa [18], e-agriculture, which requires a tight coordination between the agribusiness and professionals, including farm engineers, ICT service providers, soil analysts and e-government agencies, can hardly be implemented successfully.

As a starting point of our approach, we believe that the priority should remain on creating communication channels between farmers, allowing them to benefit from their own experience. Information from external partners need only be advertised at one end of one communication channel, the system being responsible for routing it to all participating farmers. 
Effective information : What and How? The actual synthesis of the information is another challenge : is information directed to rural populations easily extractable and readily accessible to them? These issues are indeed often overlooked in the process of designing ICT for rural areas, leaving solutions incomplete or unsuitable to the deployment context. Rural radio stations as a mass information dissemination medium is not necessarily suitable for detailing e.g. usage protocols of soil entrants, and for an on-the-need information. Nevertheless, one-to-one communication with cellular phones are still expensive resource-wise.

We believe that establishing communication channels between farmers should improve load balancing by reducing the number of communication sessions with outside support teams while leaving the possibilities for pushing relevant information for specific groups.

Reliable dissemination: How? Finally, we raise the question of how information is conveyed into and within rural areas? In the past decade, the trend has been to take advantage of the vulgarization of mobile phones as a way in the information society. This solution however has its limits: (1) cost of voice is still relatively high; (2) mobile phones, because of their "personal" aspect, do not really integrate the idea of community; (3) text messages, while a global phenomenon, are useless because of high rates of illiteracy. With the recent advances in electronics, cheap, yet powerful, devices have emerged, which can be used as handheld dashboards by farmers. These devices have capabilities for (a) one-to-one and free-of-charge connections with (b) various display modes for representing data in illiterate-friendly formats such as graphs.

We propose to rely on these devices in rural areas to seamlessly build opportunistic, delay-tolerant, networks that reflect the culture of Sub-Saharan populations and provide the means to disseminate strategic information. We discuss in the following the suitability of opportunistic networks to sub-Saharan Africa, to provide the basis for encouraging such networking solutions.

\section{Opportunity of opportunistic networking in Africa}

Opportunistic networks have lately emerged as a viable alternative networking solution for environments where the communication can afford to be challenged by sporadic and intermittent contacts as well as frequent disconnections and reconnections. In this setting, the assumption of the existence of an end-to-end path between the source and the destination is relinquished. We thus find this type of Delay Tolerant Networks to be suitable to the realities of sub-Saharan rural areas. Nonetheless, the deployment of communication systems based on such networks often face fierce criticism from skeptics who question the benefits and long-term value-added of these types of networks [8]. In this section, we revisit the targeted environment to highlight the practical issues that constitute the main barriers to the development of conventional ICT infrastructures. Subsequently we discuss various aspects of the society in this environment that can be explored for proposing a new networking scheme.

In most rural areas of sub-Saharan Africa, there is almost no ICT infrastructure [2]. Information is usually carried by travellers from the city to villages and 
across villages. Unfortunately, existing roadways accommodate only rural inhabitants who, somehow, can manage with unreliable road transportations during rainy seasons and through zones where bandits are running wild. Immediate networking solutions such as extending the operators' networks into these areas are simply economically infeasible and politically unjustifiable. In rural areas where there is some partial coverage of cellular networks, due to the limited infrastructure, it is impossible to exchange complex data requiring processing steps which may be costly in terms of bandwidth resources for conveying rich information, and computation power for displaying them. There is thus a need for exploring different networking models.

Opportunistic networks, however, present challenges, inherent to the deployment context, that must be carefully dealt with to ensure the success of the application scenarios. We discuss such challenges in the following to demonstrate how cultural models in Sub-Saharan Africa make the case for opportunistic networks. To this end we refer to the conclusions of Lindgren and Hui when they discussed the quest for a killer app for opportunistic and delay tolerant networks [8].

Technology constraints. Opportunistic networks usually rely on short range radios whose characteristics, however, also form the constraints in the design of applications scenarios. The Bluetooth technology, which is widespread in mobile devices and consumes relatively few resources, is limited in terms of the number of simultaneous paired devices. The length of discovery and setup times also constitute important limitations that can hinder the success of a solution where data transfer must remain transparent to the users' regular activities.

The impact of the first limitation is manageable since sub-Saharan rural population density is low [5]. In regular places where the studied scenario is likely to benefit from interconnection opportunities, peasants often regroup in small committees following some criteria of a culturally-defined hierarchy. Furthermore, in sub-Saharan context, members of communities regularly cross paths, leading to a reduction of discovery and connection times as their devices store information about each other.

Human dynamics. Understanding the target environment and the behavior of potential actors in an opportunistic network is vital to establish the concreteness of the opportunity for interconnecting users in a regular and efficient basis. Daily life of sub-Saharan farmers evolve around agricultural activities in remote fields and trades during market days [19]. Market places, which are generally located at the intersection of all roads leading to the village, constitute the point of arrival/departure for travellers. They also serve various purposes including village meetings [11], and can thus host a central hub for a community network.

For most rural villages in Burkina Faso, market days occur every 3 days without break and provide the opportunities to meet the other people who walk or bike from closest villages. During these days, farmers spend their time, in small groups, conversing and drinking the local beer with their acquaintances, thus creating the opportunities for exchanging data.

Participation Incentives. An application scenario for delivering communication channels through opportunistic networking is only feasible if there are incentives 
for users to sustain the network. Boot-strapping the adoption of an opportunistic application is thus the first challenge for its success. Another challenge consists in devising an application scenario that users can identify with.

The success of the microcredit phenomenon in recent years in developing regions has proved that people feel more familiar and comfortable with concepts that borrow from their own tradition [17]. In sub-Saharan Africa, people trust each other with their verbal messages or letters to forward to a recipient with the possibility for these letters to be handed over to intermediate people who may have no relationship with the initial sender nor the final addressee. Opportunistic networking will therefore fit in such a context.

Business models. An important challenge with opportunistic solutions is to ensure that they build atop a business model where it makes sense for a service provider to support them. Infrastructure costs and operating expenses can indeed constitute important barriers for the adoption of different applications.

As developed by Brewer et al., when designing solutions for developing regions, the focus must remain on shared technology, such as community kiosks, and even shared devices [4]. Sub-Saharan rural areas indeed have a history of shared technology including tractors and other hardware that are part of a cooperative's resources. The deployment and maintenance of a system for an efficient communication within the community can then also be considered as part of the services that cooperatives provide to their subscribing members.

\section{The Toolé Approach}

We discuss in this section our approach of opportunistic networking for rural areas. Rather than detailing the algorithms required for ensuring efficiency in the establishment and the management of the network, we focus on the highlevel strategies. For more information on the algorithms, we refer the reader to our previous work on the propagation of software updates in high-cost and/or low-bandwidth connectivity areas [3]. To illustrate the approach we rely on a use-case with common rural cooperatives.

\subsection{Empowering rural cooperative members}

Farmer Adama is a member of an agricultural cooperative which has members in several villages of a rural area. Regularly, whilst engaging in his daily work, Adama gathers information data on his agricultural activities (weight of harvest, local sale prices, quality assessment of products, growth of plants, etc.) and stores them in his Toolé-compliant mobile device, using a dedicated application that is illiterate-friendly. The data thus saved by Adama could benefit to other members of his cooperative for their immediate needs such as comparison of strategies, prediction of harvest, inference of best market prices. It can also be recovered and merged with data from other participants to track the evolution of the agricultural situation in a given area. Thus, a synthesis of all information must be performed consistently and transferred among cooperative members within Adama's village and into nearby villages. Because of the complexity of the data, available communication lines are unfit to properly convey the information. 
Sharing locally. As in most villages, at the end of a day of labor, Adama and his farmer friends regroup in one of the establishments that serve the traditional millet beer to discuss, thus providing the opportunities for their devices to synchronize their data through crossed communications that remain seamless and transparent to the farmers. By duplicating all data into every other farmer's device, the approach brings all farmers' devices to the same level of information, thus allowing any of them to forward the message from this village to another.

Storing reliably. Since local sharing may not enable an effective dissemination of local information into nearby villages, we resort to the widely used strategy of a black box located at the center of the village, namely the market. After the meeting where local synchronization was performed, one of the participants heads towards the marker and taps - by approaching very near - his device to the black box. This initiates a rapid transfer of all Toolé data into the box. This box, which serves as the community high-capacity database, is a "central hub" that enables a coherent merging of all distributively collected data for multiple cooperatives. It also includes a computation module for synthesizing agricultural information statistics into illiterate-friendly diagrams.

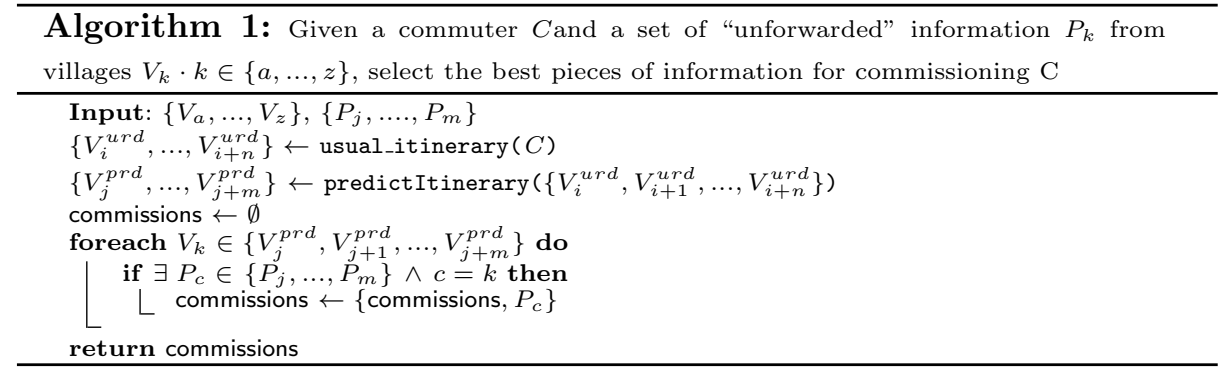

Disseminating globally. Synthesized data stored in the black box needs to be conveyed to neighboring villages to implement an epidemic propagation of cooperative data. In our approach, any farmer commuting to another village, e.g. to attend a market day, can willingly tap his phone on the black box, automatically triggering the transfer of data that the farmer will deliver to his destination black box or to someone else who will, following the culturally-established system of "commissions" in sub-Saharan Africa. It is to be noted that the black box encompasses some intelligence that allows it to track the routes of information across villages and the identities of farmers who transported the data. These traces allow for more efficiency in the select of the best traveller and the best portion of transfer data to match for commissioning. Algorithm 1 provides an overview of the strategy used by the black box to automatically choose the best data to commission a known traveller. With its perpetual learning model which allows it to reconsider evolving information, e.g., as after a bridge collapse (see Fig. 1), that redefines the paths between villages, the Toolé approach ensures that information from a given village will be widely advertised and that no village will remain isolated from the rest. 


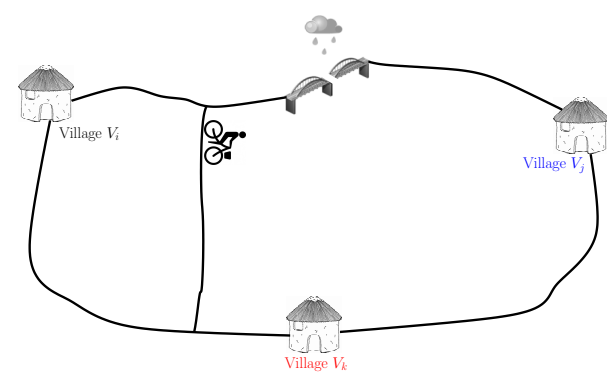

A villager $B$ is biking regularly from village $V_{i}$ to village $V_{j}$ for his trade needs. The black box in village $V_{i}$ has kept track of this travel pattern. After a change in the routines of other commuters, the algorithm "learns" that the road to Village $V_{j}$ now passes through Village $V_{k}$ (e.g., a bridge has collapsed). The black boxes forwards, into $B$ 's Toolé device, all pieces of information for Villages $V_{j}$ and $V_{k}$ as both now belong to the traveler's predicted path (cf. Algorithm 1).

Fig. 1. The Black box is an intelligent entity : Inference from environmental events

\subsection{Architecture}

We briefly highlight aspects of the architecture that we have devised to support our opportunistic scheme. It is a question of defining a system that takes advantage of the local connectivity capabilities of mobile phones to spread, in an efficient way, complex agricultural information in places where they are needed. The architecture is thus built to sustain the operation of three main processes as illustrated in Fig. 2: (1) a seamless local exchange of personal agricultural data among farmers devices; (2) an efficient and automated provision of data into the black box; and (3) a fast and reliable retrieval from the black boxes in neighboring villages.

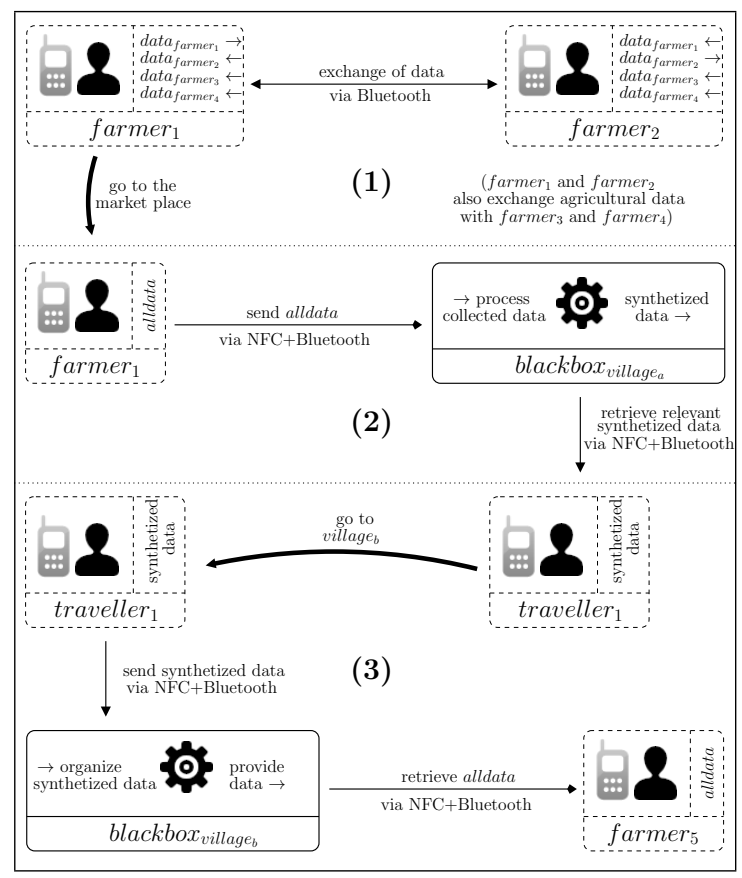

Fig. 2. The Architecture of Toolé

Bluetooth and data exchange. We resort to the Bluetooth technology for enabling a free, reliable and opportune communication between devices carried by the farmers and between devices and the black boxes. In previous sections, we have already noted the flaws of Bluetooth, especially its pairing latency and the limitations in simultaneous connections. We have discussed however, how these issues remain irrelevant because of the characteristics of rural areas.

NFC and transfer automation. The Near Field Communication technology is gaining ground as a promising technology for simplifying the triggering of radio communications [1]. We rely on the NFC standards which are increasingly 
implemented in cheap feature-devices, such as the Sagem Cosy Phone ${ }^{2}$, to automate the synchronization between Toolé devices and the black box by enabling a seamless and transparent initialization of Bluetooth connections.

\section{Related Work}

In our work effort to setup bridges between rural areas in Sub-Sahara n Africa and the Information society, we have focused on the organization of cooperatives as they presented an immediate need to access global information. Based on experiences of other researchers that attempted to improve ICT coverage in developing regions, we have taken a backwards approach and reviewed the cultural model of rural societies to establish the suitability of opportunistic networks. Related work therefore span different domains from both technical and social categories.

Cooperatives in Africa. The International Labour Office 2008 report entitled "Cooperating out of poverty" sets the tone on the omnipresence of cooperatives in Africa [13], where they represent a significant part of the private sector in most countries. Such regroupments have show an extraordinary effectiveness in improving peasants' livelihood through initiatives that are born of and reflect their own culture [15]. Nevertheless, in today's global economy, cooperative are now required to integrate themselves to the information society, a step that is complicated by the lack of ICT infrastructure.

ICT in developing regions. With the Dot-com bubble, many have seen ICT as the long-waited tool to help close the gap between industrialized countries and the developing world by allowing the latter to leapfrog into the Information economy [10]. Nevertheless the global indicators of ICT development in SubSahara n Africa are still low. Following the Internet bubble, community Internet kiosks as well as public telecenters had boomed in sub-Saharan, but have rapidly declined as interest from under-equipped users has faded [2]. The mobile phone appears today as the new archetypal application allowing users to be producers and encouraging innovations as opposed to simply consuming information. In our work, we propose a step forward to improve this innovation process by relying on more efficient networking, and by exploiting more link opportunities.

Opportunistic networking. Opportunistic networking has been successful in recent years as researchers attempt to leverage the strong patterns of encounters and movements that are involved in human daily activities. Leveraging the notion of familiar stranger [14] in big cities, Papadopouli and Schulzrinne [12] and McNamara et al. [9] have proposed content dissemination through opportunistic contacts, e.g. in urban transports. In previous work, we have proposed the Typhoon Middleware for epidemic propagation on mobile software updates based on opportunistic networking [3].

These works however remain targeted at demonstrating research state-of-theart without realistic purposes of impacting real-world scenarios. In this paper, Toolé, is directed at rural areas where peasants can actually benefit from opportunistic networking as a viable road towards integrating the information society.

\footnotetext{
2 http://www.nfcworld.com/2010/09/10/34448/hands-on-sagem-wireless-new-nfc-enabled-cosyphone/
} 


\section{Conclusion}

ICT for development has been for the past decade a prolific research field shared by technology enthusiasts, social studies scholars, and NGO members. Despite the proliferation of ideas and solutions proposed, the usage of ICT is still sparse in sub-Sahara $\mathrm{n}$ Africa. In this paper we have introduced the Toolé approach for leveraging the cultural model and traditions of sub-Saharans to build opportunistic networks. We have discussed the challenges in today's architectures and presented our approach, using a scenario with rural cooperatives. Another contribution of this paper is the discussion provided on the opportunity of opportunistic networking in Africa, to encourage the exploration of such solutions.

\section{References}

1. Near field communication in the real world - turning the nfc promise into profitable, everyday applications. Technical report, Innovision Research and Technology, 2007.

2. D. G. Alemneh and S. K. Hastings. Developing the ICT infrastructure for Africa: overview of barriers to harnessing the full power of the internet. Journal of Education for Library and Information Science, 41(1):4-16, 2006.

3. T. F. Bissyandé, L. Réveillère, Y.-D. Bromberg, and J.-R. Falleri. Typhoon: A middleware for opportunistic propagation of software updates. In $M$-MPAC, 2011.

4. E. Brewer, M. Demmer, B. Du, M. Ho, M. Kam, S. Nedevschi, J. Pal, R. Patra, S. Surana, and K. Fall. The case for technology in developing regions, may 2005.

5. P. Dorosh, H. G. Wang, L. You, and E. Schmidt. Road connectivity, population, and crop production in sub-saharan africa. Agricultural Economics, 43(1), 2012.

6. M. Harkin. ICT Adoption as an Agricultural Information Dissemination Tool - An Historical Perspective.

7. International Telecommunication Union. Measuring the information society, 2011.

8. A. Lindgren and P. Hui. The quest for a killer app for opportunistic and delay tolerant networks (invited paper). In CHANTS, pages 59-66, 2009.

9. L. McNamara, C. Mascolo, and L. Capra. Media sharing based on colocation prediction in urban transport. In MobiCom, pages 58-69, 2008.

10. N. Negroponte. The third shall be first. Wired Magazine, 6(1), 1998.

11. E. N. Obiechina. Culture, tradition and society in the West African novel. Syndics of the Cambridge University Press, 1975.

12. M. Papadopouli and H. Schulzrinne. Effects of power conservation, wireless coverage and cooperation on data dissemination among mobile devices. In MobiHoc'11.

13. I. P. Patrick Develtere and F. Wanyama. Cooperating out of poverty: The renaissance of the African cooperative movement. International Labour Office, 2009.

14. E. Paulos and E. Goodman. The familiar stranger: anxiety, comfort, and play in public places. In $C H I$, pages 223-230, 2004.

15. P. Pradervand. Listening to Africa: developing Africa from the grassroots. Greenwood Pub Group Inc, 1989.

16. S. Shakkottai and R. Srikant. Peer to peer networks for defense against internet worms. In Interperf, page 5, 2006.

17. United Nations. Microfinance in africa: Combining the best practices of traditional and modern microfinance approaches towards poverty eradication, 2000.

18. G. Weszkalnysa. The governance of daily life in africa: ethnographic explorations of public and collective services. Review of African Political Eco., 38(128), 2011.

19. L. J. Wood. The functional structure of a rural market system. Geografiska Annaler. Series B, Human Geography, 57(2):109-118, 1975. 